\title{
The Pulsed Arc Electrohydraulic Discharge Treatment Study on Waste Water and Its Application
}

\author{
Qi-Xiong LIN, Lu ZHU, Zheng-Hao HE, Wei XIA, Xin-Ya XU \\ State Key Laboratory of Advanced Electromagnetic Engineering and Technology, Huazhong University of \\ Science and Technology, Wuhan, China
}

\begin{abstract}
With the speedy development of modern industry, the technology of wastewater treatment plays an essential role in the research of environmental protection science. The tech of Pulsed Arc Electrohydraulic Discharge (PAED) has been intense researched by virtue of its marked treatment effect, low power consumption and so on. The experiment was conducted in the commercial second class waste water treatment plant by the tech of PAED. The $10 \mathrm{~L}$ volume PAED reactor with cross flow eccentric electrode arrangement was used for batch mode. The PAED power supply is $3 \mathrm{~kJ} /$ pulse with $2 \mathrm{~Hz}$ discharge rate. Two different types, one from up-stream and the other from down-stream of bio-treatment pound, with different level of $\mathrm{pH}$, electrical conductivity and E-coli concentration were treated. Three different electrode materials Ti, W and Tialloy were tested. There results show that $\mathrm{W}$ electrode showed the best performance for the inactivation of $\mathrm{E}$ coli at $1 \mathrm{kWh} / \mathrm{m} 3$ of cumulative electrical energy in-puts. Fundamental discharge characteristics, pressure wave propagation, optical emission characteristics etc. link with disinfection performance will be discussed in detail.

KEYWORD: Pulsed Arc Electrohydraulic Discharge, Discharge electrode, Wastewater treatment
\end{abstract}

\section{INSTRUCTION}

In recent years, with the sustainable development of pulsed power technology, PAED gains tremendous advancement [1-3]. PAED is the technology which utilizes the electro-hydraulic efficiency to generate the pressure wave, $\mathrm{UV}$, the $\mathrm{H}, \mathrm{O}$ and $\mathrm{OH}$ radicals [4]. The aim of killing the microbes can be realized through the physical-chemical reaction of the resultant [5-6]. For instance, the protein film of bacteria can be destroyed by pressure shock wave; the molecular bond of the bacteria's DNA also can be destroyed by photo-decomposing of UV and oxidative cracking of $\mathrm{OH}$ radicals [7-9]. Compared with the other tech of water treatment(PCED, PPED), the tech of Pulsed Arc Electrohydraulic Discharge has obvious advantages on the aspect of treatment effect energy efficiency [10].

Generally speaking, the PAED system operates at a frequency from 0.01 to $100 \mathrm{~Hz}$ with the peak current above 10kA and the voltages ranging from $2.5 \mathrm{kV}$ to $3.5 \mathrm{kV}$. After the breakdown of air switch during the process of PAED, the energy stored in the high voltage pulse capacitor can be released through the certain distance of water arc electrode gap and the violent discharge phenomenon can be formed [1112]

Research on the subject of the PAED Physicalchemical effects has been done very systematically and deeply.

N. Karpel Vel Leitner et al. observed that the highest UV intensity can be generated by PAED and the wavelength range is from $380 \mathrm{~nm}$ to $425 \mathrm{~nm}$ when the energy of the energy pulse power supply was $0.5 \mathrm{~kJ} /$ pluse [13]. B. R. Locke et al. obtained the relationship between sterilizing rate and $\mathrm{PH}$, conductivity, treatment time by PAED [14]'P Sunka et al.[15] pointed out the effect of electric field intensity on the generation rate of free group when the water arc electrode gap was Needle-Plate.

In the present article, the research on combining the tech of PAED with wastewater treatment was not so much, moreover, its main directions was the relationship between sterilizing rate and $\mathrm{PH}$, conductivity, treatment time by PAED. However, in this article the research was focused on the relations between the E. coli sterilization efficacy of PAED and the water arc electrodes. In addition, this paper also makes an analysis on the resultant after the discharge of PAED occurred. 


\section{EXPERIMENTAL APPARATUS}

The schematic diagram of experimental apparatus is shown in Fig. 1. The experimental apparatus consists of two parts: the pulsed arc power supply and reaction chamber $(10 \mathrm{~L})$. Among them, the power supply of PAED which consists of a slide transformer (AC 220V), a high voltage transformer, an energy storage capacitor $(20 \mu f \sim 160 \mu f)$, rectifier, resister $(3 \mathrm{k} \Omega)$ and a pseudo spark switch can provide a single pulse energy of $3 \mathrm{~kJ}$ and the range of its charging voltage is from $2.5 \mathrm{kV}$ to $3.5 \mathrm{kV}$. The discharge frequency of this wastewater treatment system is $0.1 \mathrm{~Hz} 100 \mathrm{~Hz}$.

The reaction chamber $(10 \mathrm{~L})$ is a stainless steel drum which has a diameter of $25 \mathrm{~cm}$. In the reaction chamber there are four positions which are used to fix the iron electrode. The distance of electrodes can be accurate control of a range from $0.5 \mathrm{~cm}$ to $1.5 \mathrm{~cm}$ by stepping motor. The four different positions are used to research on the pressure wave generated by PAED. There are six threaded holes which are located respectively on the outer end and the cylinder wall of reaction chamber. The transparent quartz pyrex window is used to observe the UV generated by PAED.

The measuring part of wastewater treatment system consists of a high voltage probe (Tektronix P6015A) which is used to measure the applied voltage, ammeter shunt (GF-1, top measure current is $30 \mathrm{kA}, 0.006879 \mathrm{~V} / \mathrm{A}$ ) which is used to measure the discharge current, and the piezoelectric type pressure sensor (CY-YD) which is used to measure the waveforms of the pressure. The voltage signals of the waveforms are sent to the electric amplifier (YE5853B) which can be displayed on the digital oscilloscope (TEK-TDS1012B) and the spectrometer is used to detect the UV generated by PAED.

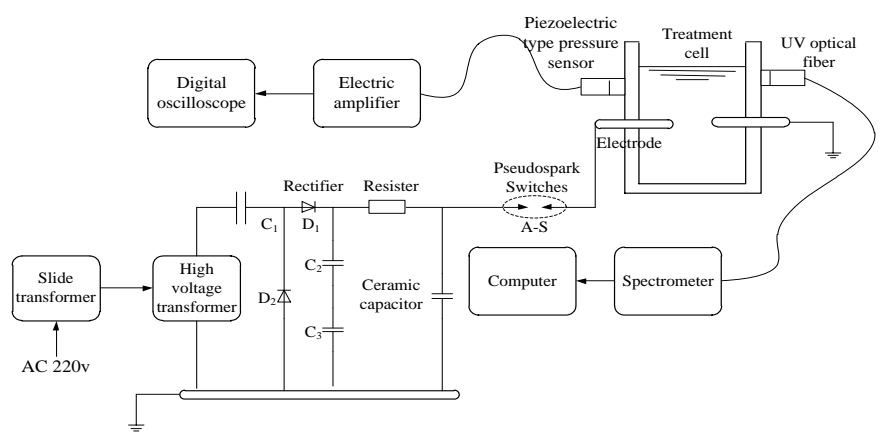

Fig.1. Schematic diagram of PAED and the analyzer system

\section{RESULTS AND DIS ICUSSION}

\subsection{The breakdown mechanism of Pulsed Arc Electrohydraulic Discharge}

After the breakdown of the plasma channel between the electrodes of pseudo spark switches, the pulse energy stored in the pulse capacitor are released into the plasma channel for a short time (about $100 \mu \mathrm{s}$ ). Than the peak current will reach dozens of $\mathrm{kA}$, the temperature of the plasma channel will also reach $10^{4} \mathrm{~K}$. With the continuous development of the plasma arc column, the water medium can be transformed into the gas. Therefore, the pulse energy has not only gasified the liquid between the two iron electrodes, but also transforms into the air bubble's internal energy and the inflation potential energy [16$18]$. With the energy transition between the internal energy and the inflation potential energy, the bubbles start to puff and shrink [19-20]. Finally, the waveforms of the pressure are formed, shown in Fig.3. The plasma channel between the iron electrodes fixed on the reaction chamber is also broken at the same time.

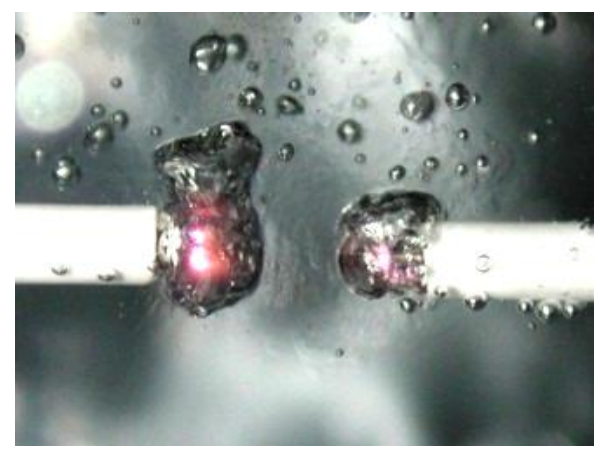

Discharge after formation of bubbles

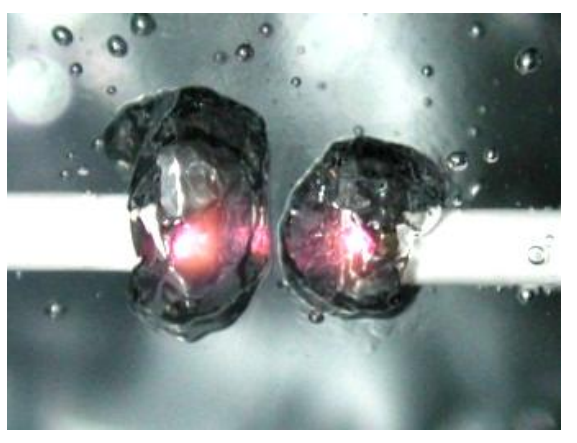

Expansion

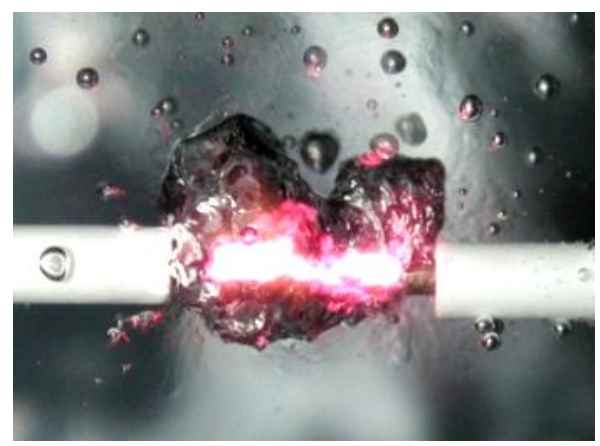

Bridging

Fig.2. Breakdown process of PAED the plasma channel

With the continuous development of plasma channel, the water medium which gets enough energy turns into the air bubble, and the formation of air 
bubble also promotes the development of plasma arc column [21] It is noticed that the discharge voltage between the iron electrodes in the water medium will not reduce to $0 \mathrm{~V}$ immediately, due to the development process of streamer channels. This kind of phenomenon can be named as the pre-breakdown of PAED. Fig. 3 shows the process of pre-breakdown lasting about $3.1 \mathrm{~ms}$. With the breakdown of the plasma channel between the pseudo spark switch, the discharge voltage will not reduce to zero. On the contrary, the discharge current will rise to the peak value in $2 \mu \mathrm{S}$. The breakdown voltage of the iron electrodes decreased from $2.5 \mathrm{kV}$ to $2.0 \mathrm{kV}$, because of the energy used to develop the plasma channel. The process of pre-breakdown is closely associated with the conductivity of water medium, PH, BOD and COD.
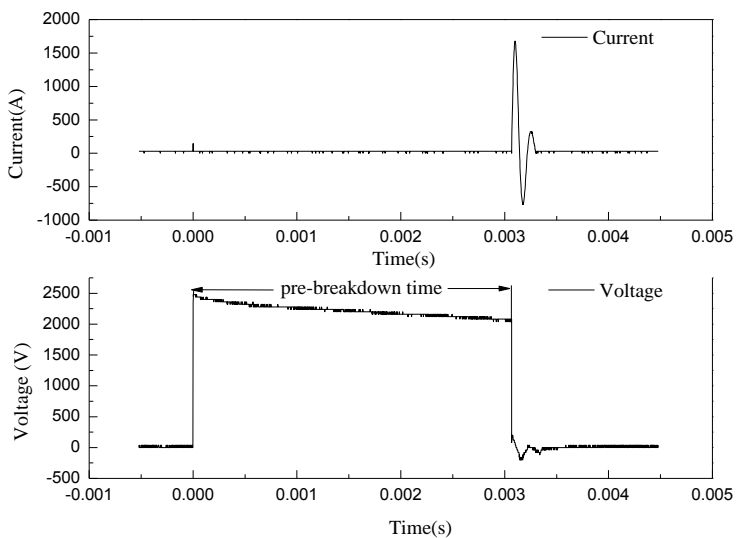

Fig.3.The voltage wave and the current wave of the water arc gap

\subsection{Relationship of the electrodes and the electric field intensity of plasma channel}

With the continuous development of plasma channel, the inter-collision of plasma, free radicals, and other free electron will be accelerated under the power of electric field. As a result, the iron electrodes and the process of PAED become closely related under the influence of electric field intensity which can be estimated by

$$
E_{\text {max }}=\frac{2 U}{r \ln \left(1+\frac{4 d}{r}\right)}
$$

In which $\mathrm{U}$ is the voltage of the iron electrodes, $\mathrm{d}$ is the distance of iron electrodes in the water medium, $r$ is the radius of the iron electrodes. Usually, the electric field intensity is closely related to the distance of iron electrodes and the radius of them. If the voltage keeps invariant, the electric field intensity will reduce along with the increase of the distance of iron electrodes. The analogous results were obtained: along with the increase ofradius of the iron electrodes, the electric field intensity will increase at first then decreases.

Fig.4 shows the simulation waveforms of the electric field intensity in the water medium. By the line of electric force between the region of the positive and the negative electrodes, it is found that the electric field intensity of the electrode border is higher than the other place, which may lead to the generation of electric arc probablelocating there.

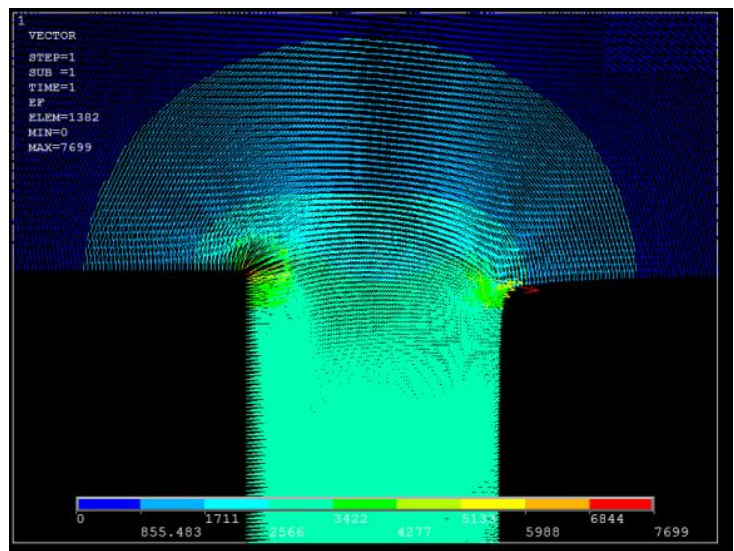

Fig.4.The simulation waveforms of the electric field intensity. The discharge voltage: $3 \mathrm{Kv}$, the distance of iron electrodes: $1 \mathrm{~mm}$, the radius of the iron electrodes: $10 \mathrm{~cm}$, the pulse energy: $3 \mathrm{KJ} /$ pulse.

\subsection{Effect of the sterilization efficacy of PAED with different distance of tungsten electrodes}

To determine the effect of the sterilization efficacy of PAED with different distance of iron electrodes, pulsed arc electrohydraulic discharge experiments were conducted at an applied. In the reaction chamber $(10 \mathrm{~L})$, the distance of electrodes changes from $0.5 \mathrm{~mm}$ to $2.5 \mathrm{~mm}$. E. coli is used as a representative to research the effect of wastewater treatment by PAED. In order to detect the number of E. coli, membrane filter method which should take a certain amount of sample $(1 \mu \mathrm{L} \sim 1000 \mu \mathrm{L})$ trained in the M-FC agar for 24hour $\left(45^{\circ} \mathrm{C}\right)$ is used. The average amount of bacterial colony which can be estimated by

$$
N=\frac{G \times 1000}{L}
$$

In which $\mathrm{N}$ is the average amount of bacterial colony (CFU/L), $\mathrm{G}$ is the average amount of bacterial colony trained on the filter, $\mathrm{L}$ is the water volume flowing through the filter $(\mathrm{ml})$.

Fig.5 (a), (b) shows the average amount of bacterial colony trained on the M-FC agar before and after discharge. The charging voltage is $3 \mathrm{kV}$, the distance of iron electrodes is $1 \mathrm{~mm}$, the pulse energy is $3 \mathrm{~kJ} /$ pulse. 


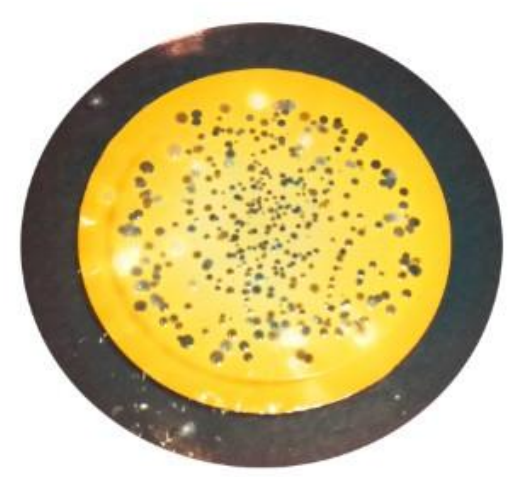

Fig.5. (a) The average amount of bacterial colony after discharge for $30 \mathrm{~min}$

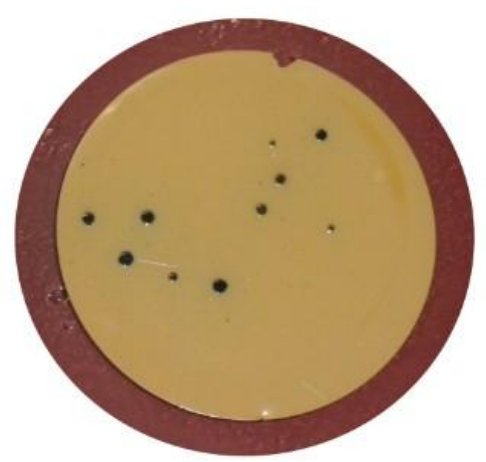

Fig.5. (b)The average amount of bacterialcolony in raw water

Fig.6 shows the effect of arc electrode gap on E. coli sterilization by PAED. Under this condition the charging voltage is $3 \mathrm{kV}$ and the conductivity of water medium is $0.688 \mathrm{~ms} / \mathrm{cm}$. Along with the increase of the distance from 0 to $1.5 \mathrm{~mm}$, the E. coli sterilization rate by PAED increases from $92 \%$ to $99.87 \%$, however, when the distance of arc electrode gap is $2.5 \mathrm{~mm}$, the E. coli sterilization rate by PAED will decrease to $56 \%$.

The reason is that according to eq.(1), along with the increase of the distance from 0 to $1.5 \mathrm{~mm}$, the electric field intensity will increase so that the initial plasma can get more energy for the electrohydraulic effect, and the effect initial plasma density will increase too. So the E. coli sterilization rate by PAED will improve. But if the distance increase to $2.5 \mathrm{~mm}$, the electric field intensity could not provide enough electric field force for the development of plasma channel, and the corona discharge will happen on the edge of the arc electrode gap. In this case, the pressure wave, $\mathrm{UV}$, the $\mathrm{H}, \mathrm{O}$ and $\mathrm{OH}$ radicals generated by PAED will reduce evidently. So the E. coli sterilization rate will decrease on $56 \%$.

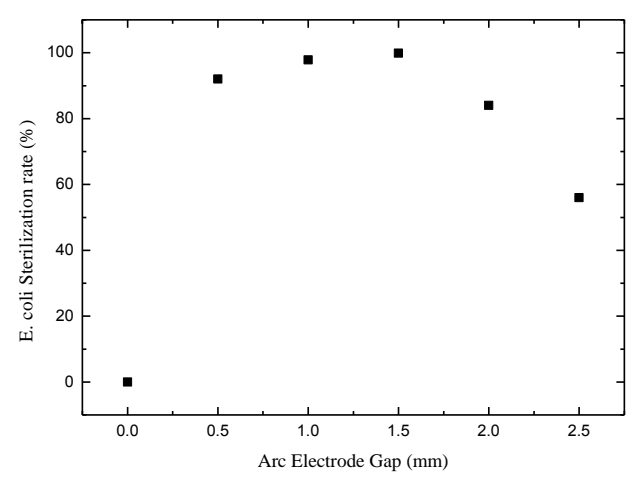

Fig.6. The effect of arc electrode gap on E. coli sterilization by PAED. Charging voltage: $3 \mathrm{KV}$, conductivity of water medium: $0.688 \mathrm{~ms} / \mathrm{cm}$, the pulse energy: $3 \mathrm{KJ} /$ pulse.

\subsection{Effect of the sterilization efficacy of PAED with different diameter of tungstenelectrodes}

Fig.7 shows the effect of arc electrode gap on E. coli sterilization by PAED. Under this condition the charging voltage is $3 \mathrm{kV}$ and the conductivity of water medium is $0.688 \mathrm{~ms} / \mathrm{cm}$. Along with the increase of the diameter from 6 to $12 \mathrm{~mm}$, the E. coli sterilization rate by PAED increases from $94 \%$ to $99.6 \%$. The reason is that according to eq.(1), along with the increase of the distance from 6 to $12 \mathrm{~mm}$, the electric field intensity will increase so that the initial plasma can get more energy for the electrohydraulic effect, and the effect initial plasma density will increase too. So the E. coli sterilization rate by PAED will improve to $99.6 \%$.

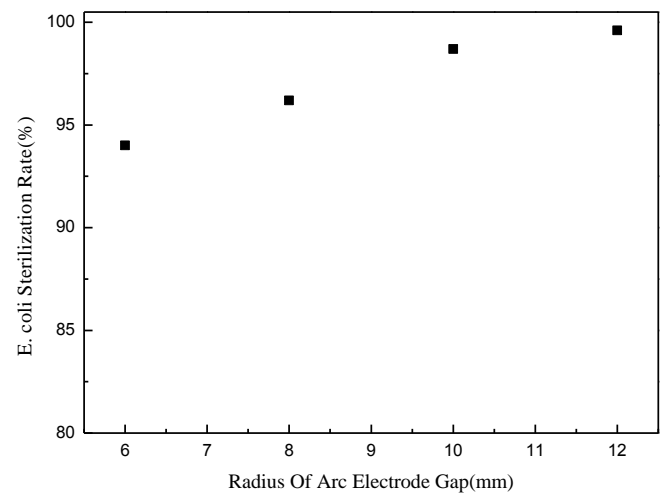

Fig.7. The effect of the radius of arc electrode gap on E. coli sterilization by PAED. Charging voltage: $3 \mathrm{KV}$, conductivity of water medium: $0.688 \mathrm{~ms} / \mathrm{cm}$, the pulse energy: $3 \mathrm{KJ} /$ pulse.

\subsection{Effect of the sterilization efficacy of PAED with different types of electrode materials}

To determine the effect of the sterilization efficacy of PAED with different types of electrode materials, pulsed arc electrohydraulic discharge experiments were conducted at an applied. Fig. 8 shows the effect 
of different types of electrode materials (Titanium, Tungsten, Titanium Alloy) on E. coli sterilization by PAED under the condition that a charging voltage reaches $3 \mathrm{kV}$ and the conductivity of water medium is $0.688 \mathrm{~ms} / \mathrm{cm}$. In this experiment, the Tungsten electrode shows better sterilization effect than that of other types of electrode materials. This is due to the secondary emission coefficient of electrode materials and fusion welding resistance are in close contact with the sterilization efficacy. The criterion for fusion welding resistance can be estimated by

$$
K=\rho C T_{M} \sqrt{\delta}
$$

In which, $\rho$ is the density of electrode materials, C is the constant of fusion welding resistance, $T_{M}$ is melting points of electrode materials, $\delta$ is conductivity. It can be noted that the melting points of electrode materials (Titanium, Tungsten, Titanium Alloy ) is respectively $1668^{\circ} \mathrm{C}, 3410^{\circ} \mathrm{C}, 1822^{\circ} \mathrm{C}$. In addition, Tungsten has the highest secondary emission coefficient among the three types of electrode materials. Therefore, with the continuous development of plasma channel, if secondary emission coefficient is higher than others, the number of high energy particles, free radicals, and other free electron will increase thus resulting in the better effect of PAED.

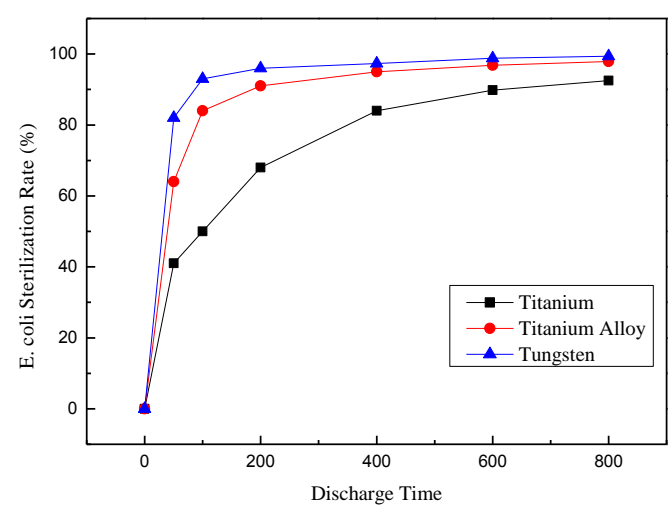

Fig.8. The effect of the types of electrode materialson E. coli sterilization by PAED

\section{CONCLUSION}

This paper states the effect of electrode in water medium by PAED. The electric field intensity of plasma channel has a close relation to the distance of iron electrodes and the radius of the iron electrodes. Along with the increase of radius of the iron electrodes, the electric field intensity will increase at first then decreases, in the meantime, the electric field intensity will reduce along with the increase of the distance of iron electrodes. With the continuous development of plasma channel, the process of prebreakdown is closely associated with the conductivity of water medium, PH, BOD and COD. Further work, effect of the E. coli sterilization efficacy of PAED is closely associated with the distance of electrodes, the diameter of electrodes and the types of electrode materials. The amount of the metal element content in water medium is the least if the Tungsten electrode is used as the electrode materials.

\section{REFERENCES}

[1] Wei C, Lin W Y, Zalnal Z. Bactericidal Activity of Tiphotocatalyst in Aqueous Media: Toward a Solar-Assisted Water Disinfection System. Environmental Science and Technology, 1994, 28(5):934-938.

[2] F. Mark. Lehr, Magne. Kristiaasen. Electrode erosion from high current arcs, IEEE Tran, on Plasma Science, 1989, 17(5): 352 355.

[3] K. H. Schoenbach, F. E. Peterkin, R. W. Alden, et al. The Effect of Pulsed Electric Fields on Biological Cells: Experiments and Applications. IEEE Trans. On Plasma Science, 1997, 25(2): 284 292.

[4] B. M. Penetrante, M. C. Hsiao, B. T. Merritt, et al. Pulsed Corona and Dielectric Barrier Discharge Processing of NO in N2. Appl. Phys. Lett. 1996, 68(26): 3719 3721.

[5] Mizuno A, Clenvents J. S, Davis R. H. A Method for the Removal of Sulfur Dioxide from Exhaust Gas Utilizing Pulsed Streamer Corona. IEEE Trans. on Industry Applications for Electron Energization, 1986, 22(3): 516 522.

[6] P. D. Pedrow, K. O. Goyal, R. Mahalingam, et al. Explosion Model Applied to an Intense Pulsed Plasma Source for Thin Film Deposition. IEEE Trans. on Plasma Science, 1997, 25(1): 89 96.

[7] Willberg D M, Lang P S, Hochemer R H, et al. Degradation of 4-chlorophenol, 3,4-dichloroaniline, and 2,4,6trinitrotoluene in an electrohydraulic discharge reactor. Environ. Sci. Technol. 1996,30: 2526-2534.

[8] Lu X, Pan Y, Liu k, et al. Spark model of pulsed discharge in water. J. appl. phys. 2002, 91: 24 31.

[9] Angeloni D M, Dickson S E, Emelko M B and Chang J-S, Removal of methyl-tert-butyl ether from water by a pulsed arc electrohydraulic discharge system. Jpn. J. Appl. Phys., 2006, 45(10b): 8290 8293.

[10] Yamatake, A., Angeloni D M, Dickson S E, Emelko M B, Yasuoka K and Chang J-S. Characteristics of pulsed arc electrohydraulic discharge for eccentric electrode cylindrical reactor using phosphate-buffered saline water. Jpn. J. Appl. Phys., 2006, 45(10b): 8298 8301.

[11]Chang J-S, Urashima K, Uchida $Y$ and Kaneda $T$, Characteristics of pulsed arc electrohydraulic discharges and their application to water treatments. J. TDDKK, 2002, 50: $1 \sim 12$.

[12]Chang J-S, Harvel G D, Yamada M, Hamada M and Okamoto Y. Ultrasonic electrical furnace electrode measurement system and methods, Japanese Patent, No. 3411192. 2003.

[13] B Legub, N. Karpel Vel Leitner, "Catalytic ozonation: a promising advanced oxidation technology for water treatment" , IEEEELSEVIER Volume 53, Issue 1, 15 October 1999, Pages 61 72 
[14]B. R. Locke, M. Sato, P. Sunka, M. R. Hoffmann, J.-S. Chang, "Electrohydraulic Discharge and Nonthermal Plasma for Water Treatment", Industrial and Engineering Chemistry Research, Ind. Eng. Chem. Res., 2006, 45 (3), pp 882 905

[15]P Sunka, V Babický, M Clupek, P Lukes, M Simek, J Schmidt and M Cernák, "Generation of chemically active species by electrical discharges in water", Plasma Sources Science and Technology, 1999, Technol. 8258

[16]C. Polk, "Biological Applications of Large Electric Fields: Some History and Funda- mentals", IEEE Trans. Plasma Sci., Vol. 28, pp, 2 14,2000.

[17] T. Namihira, S. Tsukamoto, D. Wang, S. Katsuki, R. Hackam, H. Akiyama, Y. Uchida and M. Koike, "Improvement of NO, Removal Efficiency Using Short Width Pulsed Power", IEEE Trans. Plasma Sci., Vol. 28,2000
[18] S. Katsuki, T. Majima,K. Nagata, I. Lisitsyn, H. Akiyama, M. Furuta, T. Hayashi, K. Takahashi and S. Wirkner, "Inactivation of Bacillus Stearothermophilus by Pulsed Electric Field, IEEE Trans. Plasma Sci., Vol. 28, pp. $155 \sim 160,2000$

[19]F, Hegeler and H. Akiyama, "Spatial and Temporal Distributions of Ozone After a Wire-to-Plate Streamer Discharge", IEEE Trans. Plasma Sci., Vol. 25, pp. 115\&1165, 1997.

[20]F. Hegeler and H. Akiyama, "Ozone Generation by Positive and Negative Wire-to-Plate Streamer Discharges", Japan J, Appl. Phys., Vol. 36, pp. 5335 5339, 1997.

[21] S. Tsukamoto, T. Namihira, D. Wang, S. Katsuki, H. Akiyama, E. Nakashima, A. Sato, Y. Uchida and M. Koike, "Pollution Control of Actual Flue Gas Using Pulsed Power at a Thermal Power Plant", Trans. of IEE Japan, Vol. 119-A, pp. 984 989,1999. 